\title{
In reply: Nasotracheal intubation with airway scope
}

\author{
Koyu Ono ${ }^{1} \cdot$ Tomoko Goto ${ }^{2}$
}

Received: 26 April 2014 / Accepted: 18 September 2015 / Published online: 2 February 2016

(C) Japanese Society of Anesthesiologists 2016

\section{To the Editor:}

We thank Drs. Li and Xue for their valuable comments on our study [1], and Xue and associates [2] for a description of other work in the field. The Airwayscope (AWS) provides better viewing of the glottis entrance and facilitates easier orotracheal intubation than the Macintosh laryngoscope by combining a target mark with the tube channel. These functions are not needed in nasotracheal intubation. Therefore, we did not use the target mark of the AWS in this study.

We evaluated procedural scores for difficulty during intubation, as well as the modified Cormack-Lehane grade, and the position of the AWS top blade in the vallecula or epiglottis; depending the need for external manipulation or cuff inflation. The position of the AWS blade top is listed in Table 2. There were no differences in placing the AWS in the vallecula or epiglottis between the easy, mildly difficult, or difficult intubation groups.
Finally, Xue and colleagues showed that the cuff inflation technique is effective for when the tube is excessively posterior or lateral to the glottis [2]. We agree that cuff inflation is ineffective for anterior placement of the tube tip. In the majority of our patients where the cuff inflation technique was used, the tube tip was posterior or lateral to the glottis. If nasotracheal intubation by the AWS could not be achieved, the protocol of this study called for a gumelastic bougie or bronchofiber. We did not have occasion to use a gum-elastic bougie or bronchofiber.

\section{References}

1. Ono K, Goto T, Nakai D, Ueki S, Takenaka S, Moriya T. Incidence and predictors of difficult nasotracheal intubation with airway scope. J Anesth. 2014; 28(5):650-4.

2. Xue FS, Lin JH, Liao X, Yuan YJ. Use of cuff inflation to facilitate nasotracheal intubation with the airway scope. Anaesthesia. 2011;66:754.

This comment refers to the article available at doi:10.1007/s00540-014-1897-4.

Koyu Ono

hoku4koyu3@yahoo.co.jp

1 Department of Dentistry and Maxillofacial Surgery, Itoh

Dento-Maxillofacial Hospital, 4-14 Kokaihonmachi,

Kumamoto 860-0851, Japan

2 Department of Anesthesiology, Itoh Dento-Maxillofacial

Hospital, Kumamoto, Japan 